\title{
TRES NUEVOS AGARICOMYCETES PARA LA ARGENTINA: CRUENTOMYCENA VISCIDOCRUENTA,COLLYBIOPSIS LUXURIANS Y COLLYBIOPSIS SUBPRUINOSA
}

\author{
Agustín P. Martínez ${ }^{1}$ (D) \& Bernardo E. Lechner ${ }^{1,2}$
}

\begin{abstract}
${ }^{1}$ Departamento de Biodiversidad y Biología Experimental, Facultad de Ciencias Exactas y Naturales, Universidad de Buenos Aires, Pabellón 2, Laboratorio 7, Intendente Güiraldes 2160, 1428 Ciudad Autónoma de Buenos Aires, Argentina. ${ }_{2}^{2}$ Instituto de Micología y Botánica (UBA-CONICET), Facultad de Ciencias Exactas y Naturales, Universidad de Buenos Aires, Pabellón 2, Laboratorio 7, Intendente Güiraldes 2160, 1428 Ciudad Autónoma de Buenos Aires Argentina; blechner@bg.fcen.uba.ar (autor corresponsal).
\end{abstract}

Abstract. Martínez, A. P. \& B. E. Lechner. 2021. Three new Agaricomycetes for Argentina: Cruentomycena viscidocruenta, Collybiopsis luxurians and Collybiopsis subpruinosa. Darwiniana, nueva serie 9(2): 329-341.

Three new records of Agaricomycetes are cited for the first time for Argentina: Cruentomycena viscidocruenta, Collybiopsis luxurians and Collybiopsis subpruinosa. Complete descriptions and illustrations are provided in this work, and color photographs of the fresh samples were added. Comments were made about the taxonomy and ecology of the species presented.

Keywords. Agaricales; Biodiversity; Marasmiaceae; Morphology; Mycenaceae; South America.

Resumen. Martínez, A. P. \& B. E. Lechner. 2021. Tres nuevos Agaricomycetes para la Argentina: Cruentomycena viscidocruenta, Collybiopsis luxurians y Collybiopsis subpruinosa. Darwiniana, nueva serie 9(2): 329-341.

Se citan por primera vez tres nuevas especies de Agaricomycetes para la Argentina: Cruentomycena viscidocruenta, Collybiopsis luxurians y Collybiopsis subpruinosa. En el presente trabajo se proveen descripciones completas e ilustraciones de las mismas con fotografías en color de las muestras frescas. Se realizan comentarios acerca de la taxonomía y ecología de las especies presentadas.

Palabras clave. Agaricales; América del Sur; Biodiversidad; Marasmiaceae; Morfología; Mycenaceae.

\section{INTRODUCCIÓN}

En la Argentina existe una gran diversidad de hongos Agaricales, la cual fue ampliamente estudiada por algunos micólogos relevantes para todo el mundo. En sus inicios, los estudios fueron llevados a cabo principalmente por Spegazzini, Singer y Raithelhuber, y gracias a todo el trabajo de estas personas, durante fines del siglo $\mathrm{XX}$ hasta la actualidad, se continuó trabajando con la biodiversidad de Agaricales en la Argentina. Algunos trabajos que han descripto e ilustrado nuevas especies Agaricales para la Argentina son Caffot et al. (2020),
González (2021), Kuhar et al. (2017), Lechner et al. (2003; 2004; 2005; 2006), Lechner \& Albertó (2008), Martínez et al. (2020) y Niveiro et al. $(2014 ; 2020)$.

El género Cruentomycena R. H. Petersen, Kovalenko \& O. V. Morozova (Mycenaceae, Agaricales) fue establecido por Petersen et al. (2008) y cuenta actualmente con tan solo tres especies descriptas: Cr. viscidocruenta (Cleland) R. H. Petersen \& Kovalenko, Cr. kedrovayae R. H. Petersen, Kovalenko \& O. V. Morozova, y $\mathrm{Cr}$. orientalis Har. Takah. \& Taneyama. La especie tipo de este género es $C r$. viscidocruenta, la cual anteriormente perteneció al género Mycena. 
Este género fue ampliamente estudiado en la Argentina por Singer, Raithelhuber y Spegazzini entre otros, contando con más de cien especies citadas para el país (Niveiro \& Albertó, 2012). La especie hallada y descripta en este trabajo es de gran importancia, ya que solamente existen citas en Oceanía y Sudáfrica. También es importante destacar que se trata de una especie con colores llamativos, lo cual dificulta que haya pasado desapercibida anteriormente. Petersen \& Hughes (2007) remarcaron la dispersión hacia otros continentes, mostrando el ejemplo de una colección perteneciente al género en el sureste de Rusia.

El género Collybiopsis (J. Schröt.) Earle (Marasmiaceae, Agaricales) fue restructurado por Petersen \& Hughes (2021), quienes a partir de análisis genéticos moleculares reportaron que algunas especies pertenecientes a Gymnopus sect. Vestipedes (Fr.) Antonín, Halling \& Noordel deberían agruparse para respetar el clado monofilético de Collybiopsis obtenido en sus estudios. A partir de este trabajo se modificaron los nombres de varias especies, entre ellas Collybiopsis luxurians (Peck) R. H. Petersen y Collybiopsis subpruinosa (Murrill) R. H. Petersen. Anteriormente, Niveiro \& Albertó (2013), reportaron que solamente existen unas pocas especies de Gymnopus citadas para la Argentina y Lechner (2015) reportó la primera cita de Gymnopus villosipes (Cleland) Desjardin, Halling \& B. A. Perry, perteneciente a $G$. sect. Vestipedes, y actualmente Collybiopsis villosipes (Cleland) R. H. Petersen. Collybiopsis luxurians y $C$. subpruinosa también pertenecían a $G$. sect. Vestipedes, y en el presente trabajo se citan y describen por primera vez colecciones de la Provincia de Buenos Aires, Argentina.

Durante el desarrollo de varios muestreos realizados para una tesina de grado en la reserva Municipal Los Robles, Moreno, y en otros recorridos por Provincia de Buenos Aires, se hallaron varias colecciones de tres novedosas especies para la Argentina: Cruentomycena viscidocruenta, Collybiopsis luxurians y Collybiopsis subpruinosa. El objetivo de este trabajo es reportar el primer registro del género Cruentomycena en América y dos nuevos registros de Collybiopsis para la Argentina, los cuales son descriptos e ilustrados con dibujos y fotografías a color.

\section{MATERIALES Y MÉTODOS}

Las muestras frescas fueron coleccionadas en el Partido de Coronel Brandsen y Moreno, Prov. de Buenos Aires (Fig. 1), y fueron documentadas y preservadas según la metodología estándar empleada para Agaricales (Largent, 1986). Se realizaron fotografías en campo y anotaciones acerca del aspecto antes de su secado, caracterizando macroscópicamente y teniendo en cuenta el aspecto, forma, tamaño y color del píleo, laminillas y pie; los colores se registraron de acuerdo con Kornerup \& Wanscher (1987).

Para la descripción microscópica se utilizó el método de examinación estándar para Agaricales, Wright \& Albertó (2002). Se realizaron cortes en la superficie del píleo para observar la pileipellis, en las laminillas para describir basidios, cistidios y esporas y en el pie para caracterizar caulocistidios y estipitipellis. Se utilizó el programa Piximetre para realizar la medición de todas las estructuras observadas mediante la microscopia y se utilizaron los siguientes símbolos: $\mathrm{Q}=$ coeficiente entre el largo y el ancho de las esporas, $\mathrm{Qe}=$ media de los valores de $\mathrm{Q}, \mathrm{N}=$ número de esporas medidas, $\mathrm{Me}=$ tamaño promedio de las esporas.

Se utilizaron diferentes medios y tinciones para ayudar a la observación de estructuras, ornamentaciones y reacciones sobre el basidioma fresco: $\mathrm{KOH} 3 \%$, Floxina acuosa al 1\%, y reactivo de Melzer.

Las colecciones fueron depositadas en el herbario micológico del Departamento de Biodiversidad y Biología Experimental de la Facultad de Ciencias Exactas y Naturales de la UBA (BAFC).

Para el tratamiento taxonómico se consultaron nombres y sinónimos en Index Fungorum, y descripciones de referencia para Cruentomycena (Grgurinovic, 2002; Grgurinovic \& Holland, 1982; Maas Geesteranus \& Hausknecht, 1998; Petersen et al., 2008), Collybiopsis luxurians (Antonín \& Herink, 1999; Antonín \& Noordeloos, 2010; Desjardin et al., 1999; Mata et al., 2006 [desc. luxurians var. copeyi]; Saba \& Khalid, 2014), y C. supruinosa (Desjardin et al., 1999; Song et al., 2019). También, se consultaron claves dicotómicas realizadas por Antonín \& Noordeloos (2010) y Desjardin et al. (1999). 


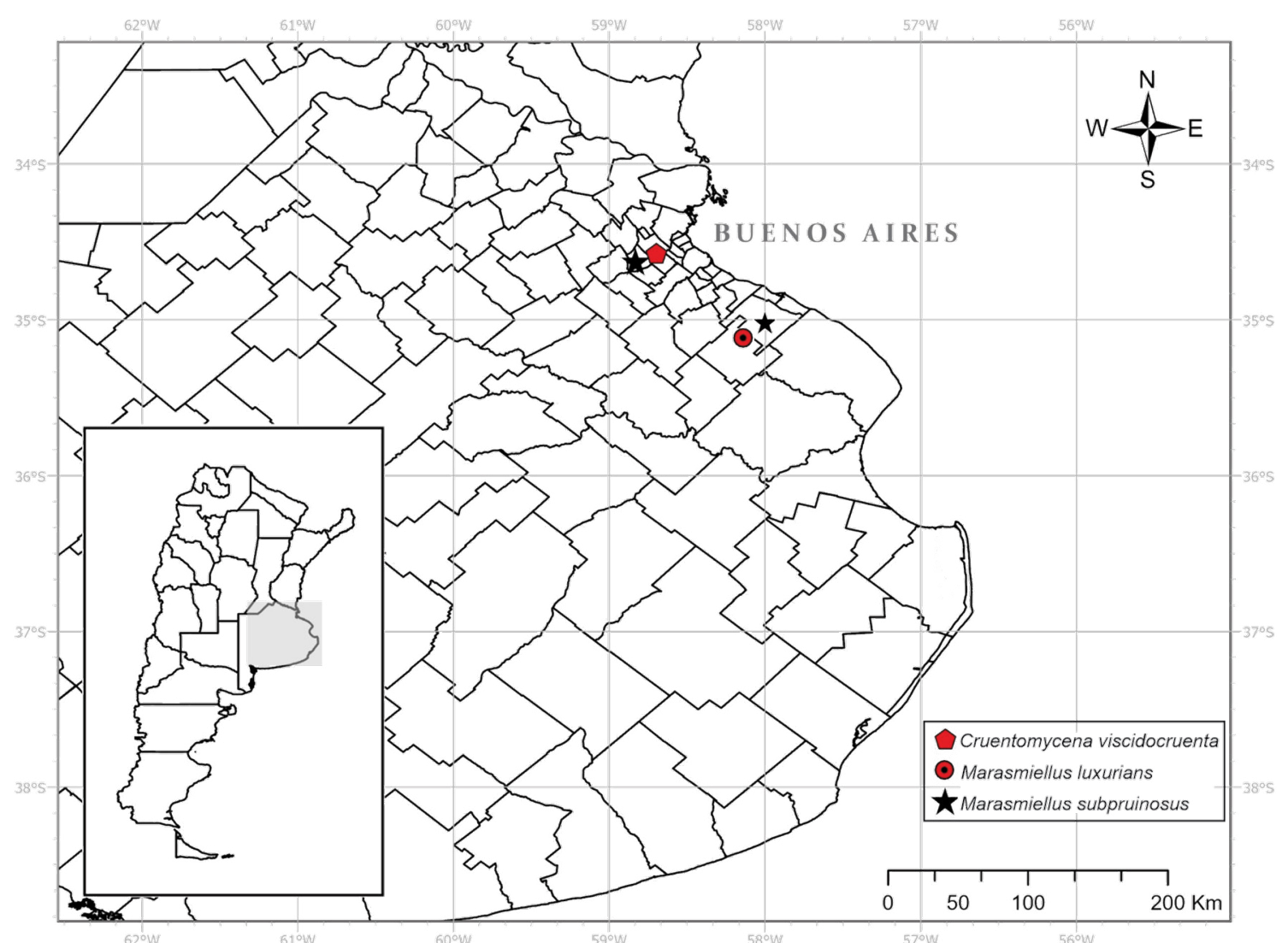

Fig. 1. Sitios de colección de los tres nuevos registros de Agaricomycetes en la provincia de Buenos Aires, Argentina.

\section{TRATAMIENTO TAXONÓMICO}

Cruentomycena viscidocruenta (Cleland) R. H. Petersen \& Kovalenko, Mycotaxon 105: 123 (2008). Mycena coccineus CLEL. \& CHEEL en Trans. Proc. Roy. Soc. S. Austral. 43: 284, figs. 2 A-D. 1919; no Mycena coccinea (SCOP.) SACC. en Flora Ital. Cryptog. 1 (Hym. 1): 276. 1915; no Mycena coccinea (SOW.) QLEL. en Bull. Soc. Amis Sei. Nat. Rouen II 15: 155 ("1879") 1880. TIPO: Mycena viscidocruenta CLELAND en Trans. Proc. Roy. Soc. S. Austral. 48: 239, pi. 29 fig. 4. 1924. Lectotipo: (design. Grgurinovic 1997): AUSTRALIA, New South Wales, Mosman, S $33^{\circ} 14^{\prime}$, E $150^{\circ} 50^{\prime}$ (precn. 3), 24.V.1914, coll. J. B. Cleland (as Mycena coccinea), det. C. A. Grgurinovic (as M. viscidocruenta), (JBC) s.n. (AD-C12182).
Píleo (Fig. 2) 4-10 mm de diám. convexo, luego convexo-aplanado, algo deprimido en el centro; viscoso a glutinoso cuando está fresco; margen crenado al madurar; superficie estriada y glabra; higrófano; coloración rojiza (10C8), con tonalidades más oscuras hacia el centro (10E8); al secarse se tornan color rojo muy intenso (10F6) a negro (10F8). Contexto escaso, de coloración rojo intenso (10D8), no exuda al corte. Laminillas adnatas a sub-decurrentes, subdistantes, con lamélulas presentes y a veces algo intervenadas; al madurar pueden llegar a separarse del pie formando un pseudocollarium; coloración más pálida que el píleo, blanco (10A2) a rosado rojizo (10A4), y el margen lamelar más rojizo (10B5); al secarse se vuelven de una coloración rojiza casi negra (10F8). Estípite 5-20 × 1-2 mm, cilíndrico, similares entre sí; viscoso a glutinoso cuando 
está fresco, brillante cuando está seco; liso y glabro, con algunas vellosidades cerca de la base; coloración rojiza intensa (10E8); libera un pigmento rojo violáceo (11A7) en $\mathrm{KOH} \mathrm{3 \% .}$ Esporada blanquecina. Olor no distintivo. Comestibilidad desconocida.

Esporas (Fig. 3a) 6,4-9,9 × 3,2-4,2 $\mu \mathrm{m}$, $\mathrm{Me}=7,4 \times 3,7 \mu \mathrm{m} ; \mathrm{Q}=(1,6) 1,8-2,3(2,6), \mathrm{Qe}=2$, $\mathrm{N}=30$; alargadas en forma de pepita y afiladas asimétricamente hacia el apéndice hiliar; pared delgada, lisas, hialinas, gutuladas, amiloides. Basidios 19,8-23,5 × 4,5-6,9 $\mu \mathrm{m}$, subcilíndricos, 4-esporados, con fíbulas. Pleurocistidios ausentes. Queilocistidios (Fig. 3b) 22, 4-36 $(42,6) \times 4,2-9,6 \mu \mathrm{m}$. cilíndricos a cilíndrico estrangulado, claviforme, esferopedunculados; algunos presentan protuberancias y morfologías irregulares, dispersos por todo el margen en gran cantidad, lisos, de pared fina, con contenido rosáceo (11A4), fibulados. Trama himenoforal irregular, compuesta por células infladas, difícil diferenciar reacción dextrinoide por la presencia de mucho pigmento rosáceo. Pileipellis un cutis, con hifas de 4-10 $\mu \mathrm{m}$ de ancho, lisas, de pared fina, no gelatinosas, fibuladas, y con contenido rosáceo (11A4), células terminales indiferenciadas, con el ápice redondeado. Caulocistidios (Fig. 3c) 34-42,2 × 7,5-12,8 $\mu \mathrm{m}$. cilíndricos a cilíndrico estrangulados, claviforme, esferopedunculados, algunos rostrados o irregulares, lisos, de pared fina, fibulados y con contenido rosáceo (11A4). Fíbulas presentes en todos los tejidos.

Hábito. Gregario o esparcido, creciendo entre ramas y hojarasca, dentro de un sendero cubierto mayoritariamente por Fraxinus, Ligustrum y Crataegus. Frecuente en épocas de mucha lluvia y humedad.

Distribución y hábitat. Esta especie se encuentra principalmente en el sudeste australiano, Queensland, Tasmania, y Nueva Zelanda (Maas Geesteranus \& Hausknecht, 1998; Grgurinovic, 2002). Recientemente fue registrada en Sudáfrica (Kinge et al., 2020). Los distintos registros muestran asociaciones no especificas con distintas especies de Cyathea, Eucalyptus y Nothofagus.

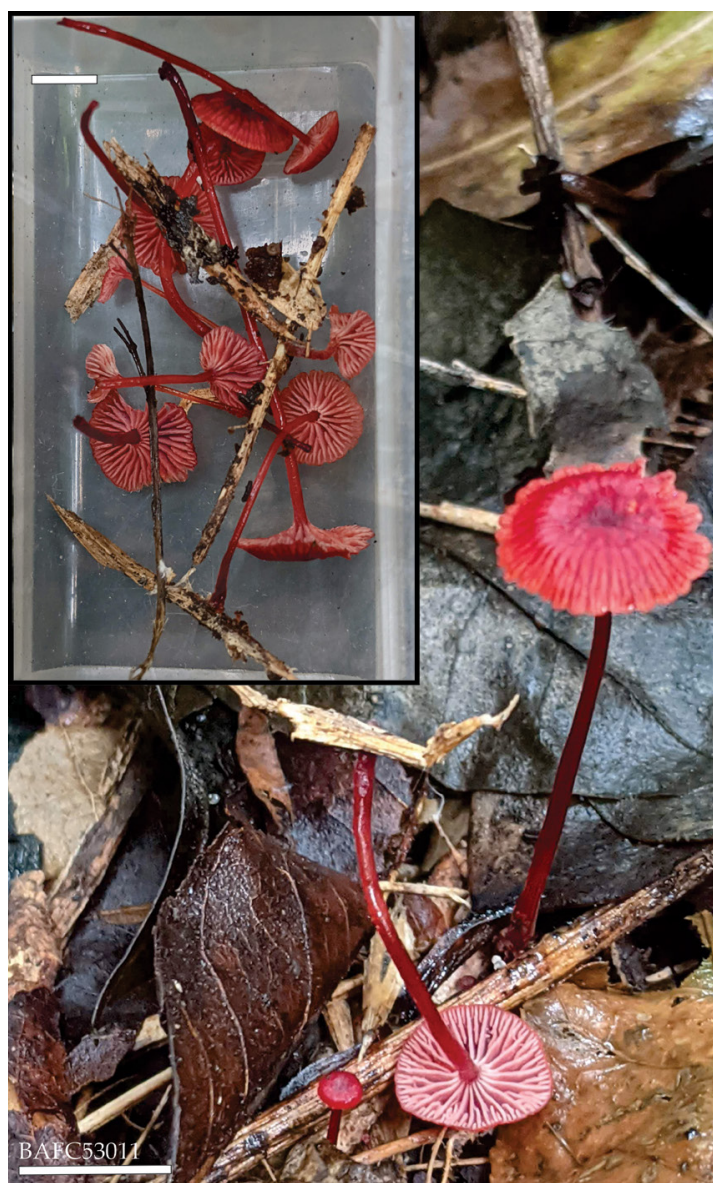

Fig. 2. Cruentomycena viscidocruenta. Aspecto general. Escala: $8 \mathrm{~mm}$. Fotografías Agustín P. Martínez. Figura en color en la versión en línea http:/www.ojs.darwin.edu.ar/index.php/darwiniana/ article/view/966/1233

Observaciones. Cruentomycena viscidocruenta no ha sido reportada en América hasta ahora. Este nuevo registro amplia la distribución global del género. Las características principales de este género son los basidiomas micenoides a marasmielloides, de coloración rojo sangre, píleo víscido, esporas amiloides, queilocistidios hialinos y la ausencia de pleurocistidios (Petersen et al., 2008). Sin embargo, nuestras colecciones y las reportadas por Maas Geesteranus \& Hausknecht (1998) y Grgurinovic \& Holland (1982) presentaron queilocistidios con contenidos de pigmento rosáceo. 


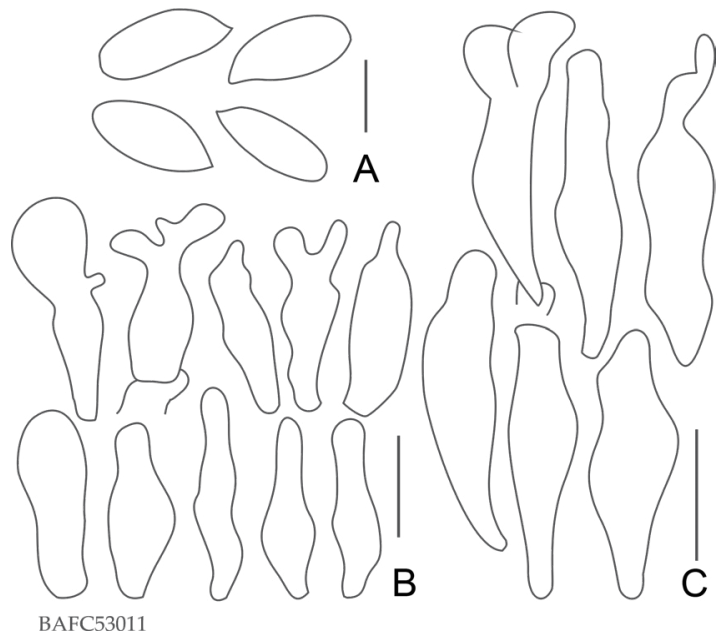

Fig. 3. Cruentomycena viscidocruenta. A, esporas (escala: $3 \mu \mathrm{m}$ ). B, queilocistidios (escala: $15 \mu \mathrm{m}$ ). C, caulocistidios (escala: $20 \mu \mathrm{m})$. Ilustraciones Agustín P. Martínez.

A partir de la microscopia de las colecciones estudiadas, se observó que los valores para el tamaño de las esporas coincidían con los rangos citados previamente por Cleland \& Cheel (1919) como Mycena coccinea, además de Maas Geesteranus \& Hausknecht (1998) y Petersen et al. (2008), aunque Grgurinovic (2002) y Grgurinovic \& Holland (1982) reportaron rangos con valores mayores, 7,2-11,8 $\times 3,4-4,5 \mu \mathrm{m}$ y $6,8-10,9 \times 3,4-5,1 \mu \mathrm{m}$, respectivamente. El tamaño y morfología de los queilocistidios observados coincide con lo citado por Maas Geesteranus \& Hausknecht (1998), aunque Grgurinovic \& Holland (1982) y Petersen et al. (2008) no registraron morfologías irregulares o con algunas protuberancias. Petersen et al. (2008) reportaron características de los caulocistidios, las cuales coinciden ligeramente con nuestra colección, donde se observaron caulocistidios de menor longitud.

Dentro del género existen actualmente tres especies descriptas: Cruentomycena viscidocruenta, Cr. kedrovayae, y Cr. orientalis. Estas especies no presentan características macromorfológicas que las distingan fácilmente; sin embargo, si existen algunas diferencias macro- y micromorfológicas entre sí. Cruentomycena kedrovayae fue descripta por primera vez a partir de una colección de
Rusia, y se distingue principalmente por tener una textura menos víscida en el píleo y estípite, característica que pareciera no depender de las condiciones ambientales, presentar un solo tipo de queilocistidios, esporas levemente más anchas y crecer específicamente bajo Alnus sp. (Petersen et al., 2008). También, mediante el estudio de una colección de Cr. kedrovayae de Corea, se agregó a la descripción de esta especie la presencia de pleurocistidios (Cho et al., 2018). Cruentomycena orientalis fue descripta por primera vez a partir de una colección de Japón, y se diferencia principalmente por la morfología de sus cistidios, los cuales se distinguen por ser mayoritariamente clavados a subcilíndricos, y algunos con un ápice obtuso. También, difieren las medidas citadas con las observadas en nuestras colecciones, que presentaron queilocistidios y caulocistidios más delgados (Terashima et al., 2016). Recientemente, en México, Cortés-Pérez et al. (2019) reportaron que Mycena guzmanii Cortés-Pérez, Desjardin \& B.A. Perry se encuentra relacionada filogenéticamente con el género Cruentomycena, presentando un $87,2-87,6 \%$ de similitud con la región ITS de varias secuencias del género.

\section{Material estudiado}

ARGENTINA, Buenos Aires, Munic. Moreno, Reserva Los Robles, 34 39' 43,2”'S 58 51'44,3” O, 2-II-2021, A. P. Martinez, BAFC 53011.

Collybiopsis luxurians (Peck) R.H. Petersen, in Petersen \& Hughes, Mycotaxon 136(2): 342 (2021). Collybia luxurians Peck, Bulletin of the Torrey Botanical Club 24: 141; (1897). Collybidium luxurians (Peck) Murrill, Mycologia 3 (3): 169; (1911). Gymnopus luxurians (Peck) Murrill, N. Amer. Fl. (New York) 9 (5): 362; (1916). Collybia compresipes Bigelow, Rhodora 78: 124. (1976). Gymnopus luxurians var. copeyi J. L. Mata \& R. H. Petersen, in Mata, Hughes \& Petersen, Sydowia 58 (2): 287; (2006). Gymnopus luxurians var. luxurians (Peck) Murrill, N. Amer. Fl. (New York) 9 (5): 362 (1916). Marasmiellus luxurians (Peck) J. S. Oliveira, Mycol. Progr. 18 (5): 735, (2019). TIPO: U.S.A., Alabama, July 1896, Underwood $\&$ Earle (NYS, holotipo). 


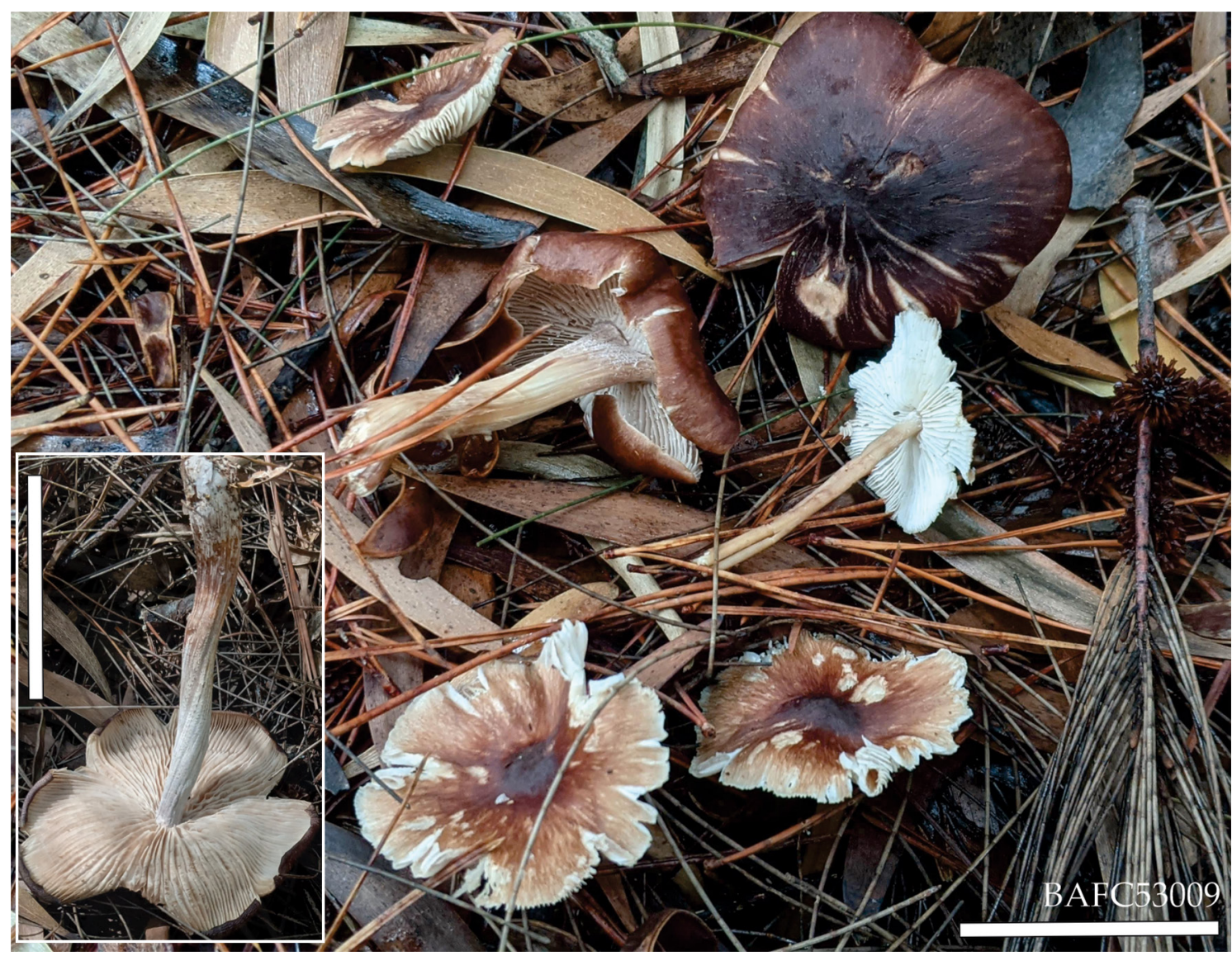

Fig. 4. Collybiopsis luxurians. Aspecto general. Escala: $30 \mathrm{~mm}$. Fotografías Agustín P. Martínez. Figura en color en la versión en línea http://www.ojs.darwin.edu.ar/index.php/darwiniana/article/view/966/1233

Píleo (Fig. 4) 15-50 mm de diám., convexo a subcampanulado, levemente umbonado, luego aplanado a cóncavo, margen curvado o levantado al madurar, no viscoso, a veces ligeramente higrófano; superficie del píleo removible, delgada, de aspecto fibrilloso castaño rojizo oscuro (8E7) cuando es joven, luego el margen se decolora a un castaño rojizo claro (8D6) a naranja rojizo $(8 \mathrm{C} 5)$, generando un aspecto algo veteado. Contexto delgado, menor a $5 \mathrm{~mm}$, blanco amarillenta (8A2) oscureciéndose hacia base del estípite. Laminillas adnexas, cercanas a sub-distantes, con lamelulas, blancas (8A1) al principio, luego grisáceo pálida (8B2) en la madurez. Estípite 10-40 × 2-5 mm, central, hueco, ligeramente engrosado en la base, longitudinalmente estriado y a veces retorcido, ápice parduzco pálido (7A3) ligeramente pruinoso, castaño parduzco (7B4) hacia la base, micelio basal blanco (7A1), generalmente formando rizomorfos. Esporada blanca a amarillo pálida. Olor no distintivo; sabor no distintivo o levemente amargo. Comestibilidad desconocida.

Esporas (Fig. 5a) $(5,8) 6,5-8,1 \times 3,2-4,2 \mu \mathrm{m}$; $\mathrm{Me}=7,2 \times 3,5 \mu \mathrm{m} ; \mathrm{Q}=1,8-2,4 ; \mathrm{Qe}=2,1 ; \mathrm{N}=35$; elipsoides a subcilíndricas, o sublacrimoide, de pared fina, lisas, inamiloide, no dextrinoide, hialinas en $\mathrm{KOH} \mathrm{3 \%}$. Basidios 5,2-6,3 × 19,427,3 $\mu \mathrm{m}$, clavados, 2- o 4-esporados, hialinos, de pared fina, esterigma 2-3 $\mu \mathrm{m}$ de largo. Pleurocistidios ausentes. Queilocistidios (Fig. 5b) 14,8-34 × 4,5-9,2 $\mu \mathrm{m}$, formas variables, cilíndricos a clavados, generalmente irregulares, de pared delgada, lisos, hialinos, a veces con el ápice lobulado o con protuberancias. Trama himenoforal 
entrelazada, compuesta por hifas de 6-9 $\mu \mathrm{m}$ de largo, cilíndricas a subinfladas, lisas, de pared fina, no gelatinosas, hialinas. Pileipellis un cutis, compuesto por hifas cilíndricas de $6-14 \mu \mathrm{m}$, lisas o algunas con incrustaciones anulares castaño parduzco (8E6), de pared delgada, elementos terminales suberectos, de 10,6-34,1 × 5,2-9,8 $\mu \mathrm{m}$, cilíndricos a clavados. Estipitipellis un cutis, hifas cilíndricas de 4-9 $\mu \mathrm{m}$, hialinas. Caulocistidios (Fig. 5c) 18,7-60,1 × 4,4-6,3 $\mu \mathrm{m}$, cilíndricos, de pared delgada, hialinos, numerosos. Fíbulas presentes en todos los tejidos.

Hábito. Dispersos a gregarios, sobre hojarasca y restos de madera en descomposición, bajo Casuarina sp., coníferas y árboles caducifolios.

Distribución y hábitat. Esta especie tiene distribución casi cosmopolita, se ha encontrado en países del Este de Asia, África Central, EE.UU., Hawái (Desjardin et al., 1999; Halling, 1983), Alemania, Italia (Hausknecht \& Zuccherelli, 1998; Contu \& La Rocca, 1999), Republica Checa, Francia (Bon \& Massart, 1996), Holanda, España, Suiza (Antonín \& Noordeloos, 2010), Costa Rica, Panamá (Mata \& Ovrebo, 2009), Republica Dominicana (Mata et al., 2006), y Pakistán (Saba \& Khalid, 2014).

Observaciones. Collybiopsis luxurians se caracteriza por su píleo de color castaño rojizo oscuro, convexo, radialmente fibrilloso, que al madurar se decolora hacia los márgenes; laminillas cercanas a subdistantes, color beige; y un estípite de color blanco a castaño parduzco, llamativamente retorcido-estriado. Desjardin et al. (1999) describieron una colección de las islas hawaianas encontrada frecuentemente en bosques de Casuarina, un ambiente semejante dónde fueron encontradas las colecciones de este trabajo. Collybiopsis luxurians solo ha sido observado asociado con plantas introducidas dentro de Hawái, por lo cual consideran que se trata de una especie introducida como "pasajera" que se extiende desde el Norte de América hacia el sur y Europa (Desjardin et al. 1999). Además, Mata et al. (2006) demostraron que las colecciones de C. luxurians de Europa, América del Norte y República Dominicana pertenecían todas a una misma especie biológica.

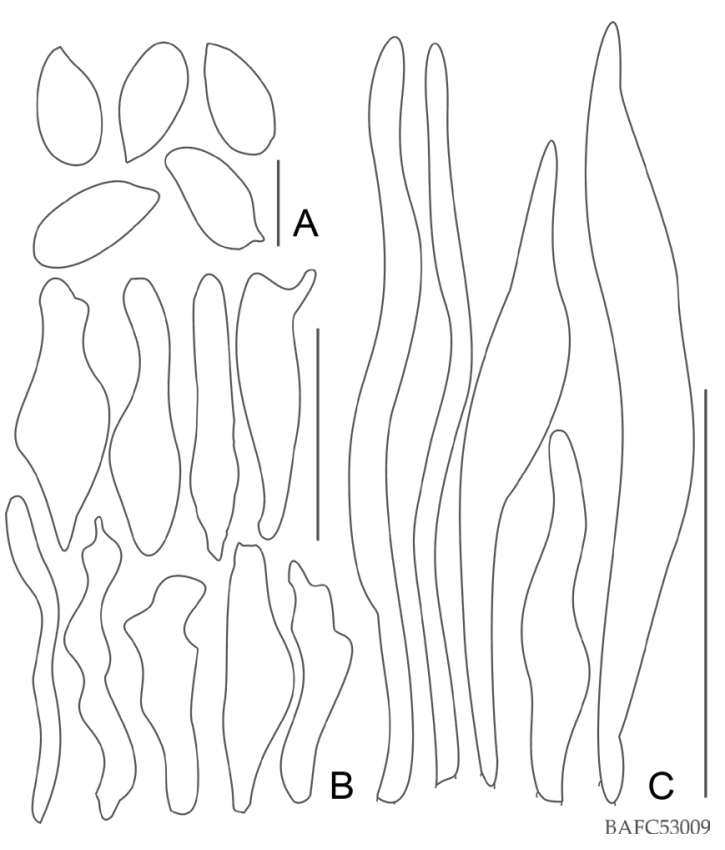

Fig. 5. Collybiopsis luxurians. A, esporas (escala: $4 \mu \mathrm{m}$ ). B, queilocistidios (escala: $25 \mu \mathrm{m}$ ). C, caulocistidios (escala: $30 \mu \mathrm{m}$ ). Ilustraciones Agustín P. Martínez.

A partir de las colecciones observadas, se obtuvo un tamaño de esporas que difiere respecto al citado por algunos autores, quienes reportaron esporas de mayor longitud: 7,0-11,0 $\times$ 3,5-5,5 $\mu \mathrm{m}$ (Antonín \& Noordeloos, 2010; Antonín \& Herink, 1999). Los valores de las colecciones obtenidas si coindicen con los rangos citados por Saba \& Khalid (2014) y Hausknecht \& Zuccherelli (1998), 4,2-5 × 5,3$9,4 \mu \mathrm{m}$ y $6,4-9 \times 3,5-4,2 \mu \mathrm{m}$, respectivamente. Los queilocistidios observados coinciden con la morfología descripta por todos los autores mencionados anteriormente, aunque el tamaño coincide solamente con el reportado por Saba \& Khalid (2014), el resto de los autores reportaron queilocistidios de mayor longitud. De manera contraria, los caulocistidios no comparten el mismo tamaño citado por Saba \& Khalid (2014), quienes reportaron caulocistidios de una longitud mucho mayor, 53-124 ×9-14 $\mu \mathrm{m}$.

Una de las especies citadas para la región con la que puede confundirse es Rhodocollybia butyracea (Bull.) Lennox. Esta se diferencia por tener esporas dextrinoides y cianófilas, 
solamente basidios 4-esporados, y por tener un pie liso, no retorcido (C. luxurians se destaca por su pie retorcido), esponjoso y con un bulbo hacia la base. Además, R. butyracea se encuentra generalmente bajo Pinus sp. y presenta un olor diferente, fuerte y rancio (Desjardin et al., 2015). Otra especie similar pero no citada para la Argentina es Rhodocollybia prolixa (Fr.) Antonín $\&$ Noordel., aunque se diferencia principalmente por tener esporas más pequeñas, 4,5-6 × 3,24,4 $\mu \mathrm{m}$ (Mata et al., 2004).

\section{Material estudiado}

ARGENTINA, Buenos Aires, Coronel Brandsen, $35^{\circ} 8^{\prime}$ 49,4" S 58० 2' 51,5" O, 6-V-2021, B. E. Lechner \& A. P. Martínez, BAFC 53009; Coronel Brandsen, $35^{\circ} 8^{\prime}$ 50,8" S 58 2' 50,8” O, 6-V-2021, B. E. Lechner \& A. P. Martínez, BAFC 53010.

\section{Collybiopsis subpruinosa (Murrill) R.H.} Petersen, in Petersen \& Hughes, Mycotaxon 136(2): 344 (2021). Basiónimo: Marasmius subpruinosus Murrill, N. Amer. Fl. (New York) 9(4): 266 (1915). Sinónimos - Collybia subpruinosa (Murrill) Dennis, Trans. Br. mycol. Soc. 34(4): 449 (1951). Gymnopus subpruinosus (Murrill) Desjardin, Halling \& Hemmes, Mycologia 91(1): 171 (1999). Marasmiellus subpruinosus (Murrill) J. S. Oliveira, Mycol. Progr. 18 (5): 735 - Figs. 43-44; (2019). TIPO: Troy and Tyre, Cockpit Country, Jamaica, 1214-I-1909, W. A. Murrill \& W. Harris 947 (herb. N. Y. Bot. Gard.).

Píleo (Fig. 6) 10-40 (60) mm de diám.; convexo a campanulado, un poco deprimido en el centro, y a veces un poco umbonado, luego aplanado al madurar y los márgenes se curvan hacia arriba; superficie lisa, seca; higrófano, coloración castaño ocrácea (5C6) a beige (5B4), estriado radialmente hacia el centro con tonalidades ocráceas más oscuras (5D7). Contexto muy delgado, menor a $2 \mathrm{~mm}$; inmutable. Laminillas adnexas a adnatas, subdistantes, con series de 3-4 lamélulas, a veces con anastomosis; margen pruinoso, coloración ocre pálida (5B3) a beige grisáceo (5A3). Estípite 10-50 × 1-3 mm, cilíndrico, central, flexible; superficie seca, ápice de coloración similar a las laminillas, ocre pálido (5A2), obscureciendo hacia la base, castaño ocráceo (5C3). Esporada blanca. Olor fúngico suave; sabor no distintivo. Comestibilidad desconocida.

Esporas (Fig. 7a) 6,4-8,2 (9,3) × 3,9-5 (5,4) $\mu \mathrm{m}$; $\mathrm{Me}=7,4 \times 4,5 \mu \mathrm{m} ; \mathrm{Q}=1,5-1,8(2,1) ; \mathrm{Qe}=1,7$; $\mathrm{N}=56$; elipsoides a fusiformes o amigdaliformes, lisas, de pared fina, hialinas, gutuladas, inamiloides, no dextrinoides. Basidios 25,2-34,8 $\times$ 7-8,1 $\mu \mathrm{m}$, claviformes, 4-esporados, gutulados. Pleurocistidios ausentes. Queilocistidios (Fig. 7b) 21,2-47,6 × 4,6-11,9 $\mu \mathrm{m}$, variables, cilíndricos a clavados, esferopedunculados o rostrados, lisos, de pared fina, hialinos. Trama himenoforal entrelazada, compuesta por hifas de 5-10 $\mu \mathrm{m}$ de largo, cilíndricas, lisas, de pared fina, no gelatinosas, hialinas, inamiloides y no dextrinoides. Pileipellis un cutis compuesto por hifas cilíndricas de 3-10 $\mu$ m de ancho, subparalelas a entretejidas, de pared fina y algunas con incrustaciones en forma de cebra, con células terminales erectas a suberectas, de 11,3-39,8 $\times$ 4,3-9 $\mu \mathrm{m}$, cilíndricas, de pared fina, superficie lisa, hialinas, algunas con depósitos de pigmentos castaño ocráceo (5D6) en $\mathrm{KOH} 3 \%$. Estipitipellis un cutis con abundantes caulocistidios, compuesto por hifas cilíndricas de 4-10 $\mu \mathrm{m}$ de ancho, lisas, de pared fina, no gelatinosas, inamiloides. Caulocistidios (Fig. 7c) 32-68,2 × 4-8,1 variables, cilíndricos a sinuosos, solitarios o agrupados. Superficie lisa, de pared fina, hialina, inamiloide. Fíbulas presentes en todos los tejidos.

Hábito. Dispersos a gregarios, sobre humus, hojarasca o restos de madera en descomposición. Frecuente durante gran parte del año, de primavera a otoño, mayoritariamente en otoño y épocas de mucha lluvia. Los basidiomas tardan mucho tiempo en descomponerse y no son consumidos por la fauna del lugar.

Distribución y hábitat. Esta especie se encuentra en el Este de EE.UU. y Hawái (Desjardin et al., 1999); en Costa Rica, Jamaica, Panamá, y Puerto Rico (Murrill, 1915; Ovrebo, 1996; Mata \& Ovrebo, 2009); en Brasil, Chile y Ecuador (Coimbra, 2017; Ortiz et al., 2014; Rosa \& Capelari, 2009); y en la región Macaronesica y Nueva Zelanda (Song et al., 2019). 


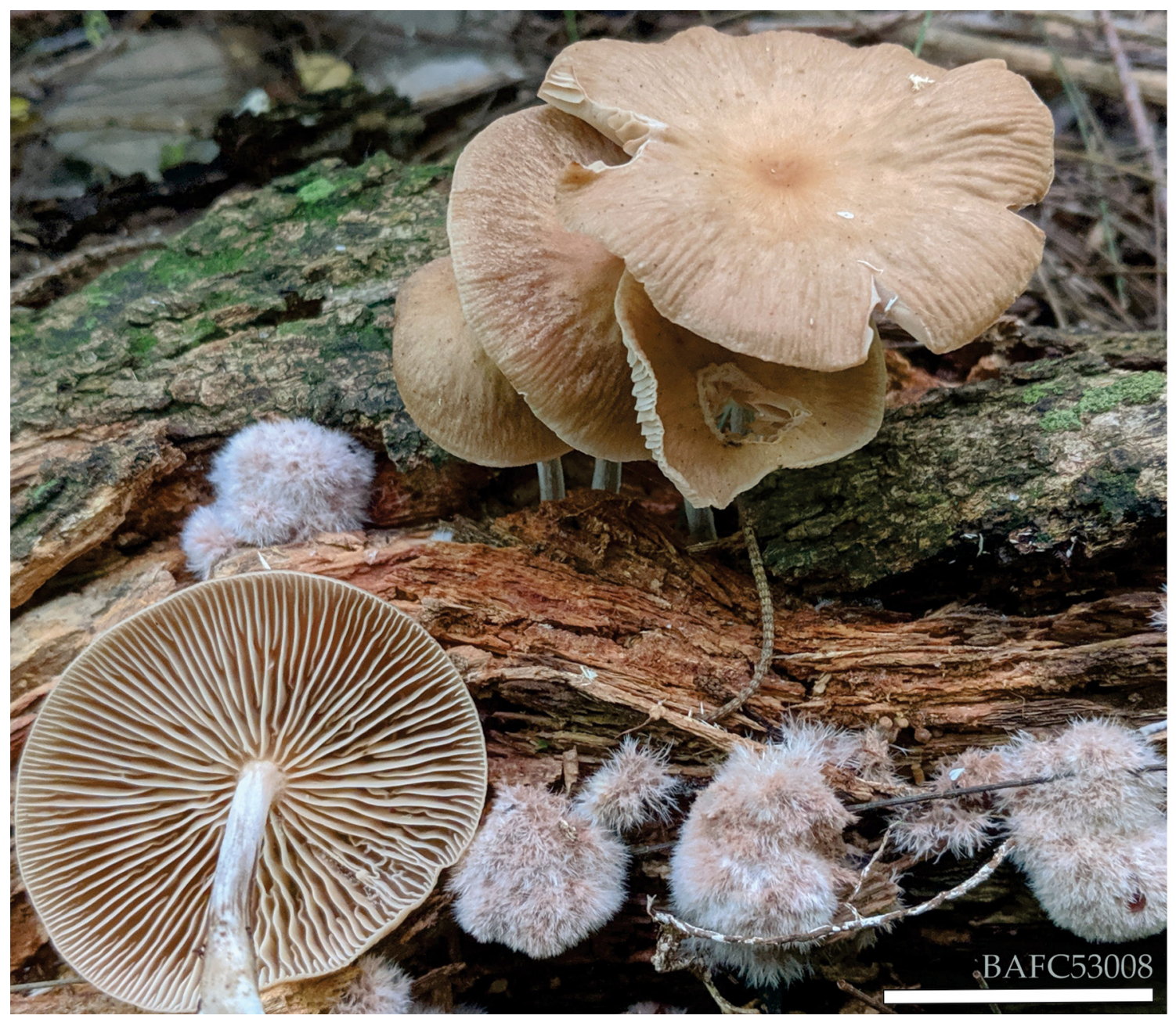

Fig. 6. Collybiopsis subpruinosa. Aspecto general. Escala: $15 \mathrm{~mm}$. Fotografía Agustín P. Martínez. Figura en color en la versión en línea http://www.ojs.darwin.edu.ar/index.php/darwiniana/article/view/966/1233

Observaciones. Collybiopsis subpruinosa se caracteriza por su píleo estriado, translucido y con tonalidades ocre pálidas, laminillas color beige y un pie delgado con vellosidades y una coloración similar a las laminillas, oscureciéndose hacia la base. Collybiopsis subpruinosa se describió originalmente basándose en especímenes jamaiquinos recolectados por Murrill (Murrill, 1915). Luego, Desjardin et al. (1999) describieron e ilustraron de manera completa ejemplares de las islas hawaianas, también se señalaron registros para California. Song et al. (2019) describieron esta especie macro- y microscópicamente, hicieron análisis genéticos moleculares $\mathrm{y}$ señalaron que $C$. subpruinosa probablemente sea una especie nativa de la laurisilva de Madeira, donde se la halló creciendo cerca de árboles endémicos de Oxydendrum arboretum (L.) DC.

Todas las colecciones observadas presentaron una morfología y tamaño de las esporas que comparten los rangos de valores citados previamente por Desjardin et al. (1999) y Song et al. (2019). El tamaño de los queilocistidios observados es menor al citado por Desjardin et al. (1999) para las 
colecciones de Hawái $(25-80 \times 5-16 \mu \mathrm{m})$, aunque coincidió con los valores citados por Song et al. (2019). También difirió el tamaño de los caulocistidios, el cual es menor al citado por Desjardin et al. (1999), 60-120 × 2,5-10 $\mu \mathrm{m}$, y nuevamente similar al citado por Song et al. (2019), 24-75 × 5,5-10 $\mu \mathrm{m}$. Además, se observaron elementos terminales de la pileipellis de morfología y tamaño similar a las colecciones de Song et al. (2019).

Algunas de las especies similares con las cuales se la pueda confundir son Collybiopsis villosipes y Gymnopus brassicolens (Romagn.) Antonín \& Noordel., aunque esta última no está citada para la Argentina. Collybiopsis villosipes fue registrado por primera vez en el país por Lechner (2015), hallado en pinares del Partido de la Costa de Buenos Aires. Esta especie se distingue por tener una coloración castaña mucho más oscura, generalmente ser umbonado y un estípite densamente tomentoso, microscópicamente se diferencia por sus queilocistidios cilíndricos y apicalmente lobulados, y la presencia de hifas diverticuladas en la pileipellis. Además, su crecimiento es exclusivo sobre hojarasca de Pinus sp. Gymnopus brassicolens no se encuentra citado para la Argentina, pero es fácilmente distinguible debido a su fuerte olor desagradable, similar al repollo podrido o ajo (Desjardin et al., 2015). Otra especie similar que no se encuentra citada para la Argentina es Gymnopus rodhallii Desjardin \& B. A. Perry, que fue descripta en Santo Tomé, África, y se diferencia por tener un píleo de coloración gris parduzca que se decolora a crema hacia el centro, laminillas cercanas, esporas más pequeñas $(6,4-7,0 \times 3,0$ $3,5 \mu \mathrm{m})$ y una pileipellis con células terminales indiferenciadas (Desjardin \& Perry, 2017).

\section{Material estudiado}

ARGENTINA, Buenos Aires, Coronel Brandsen, $35^{\circ} 8^{\prime}$ 50,2” S 58 2' 50,7 “ O, 6-V2021, B. E. Lechner \& A. P. Martínez, BAFC 53008; Munic. Moreno, Reserva Los Robles, $34^{\circ} 40^{\prime} 4,8^{\prime \prime} \mathrm{S} 58^{\circ} 51^{\prime}$ 39,6" O, 2-II-2021, A. P. Martinez, BAFC 53006; Reserva Los Robles, $34^{\circ} 40$ ' 8,4" S 58 51' 43,2" O, 19-IV-2021, A. P. Martínez, BAFC 53007.

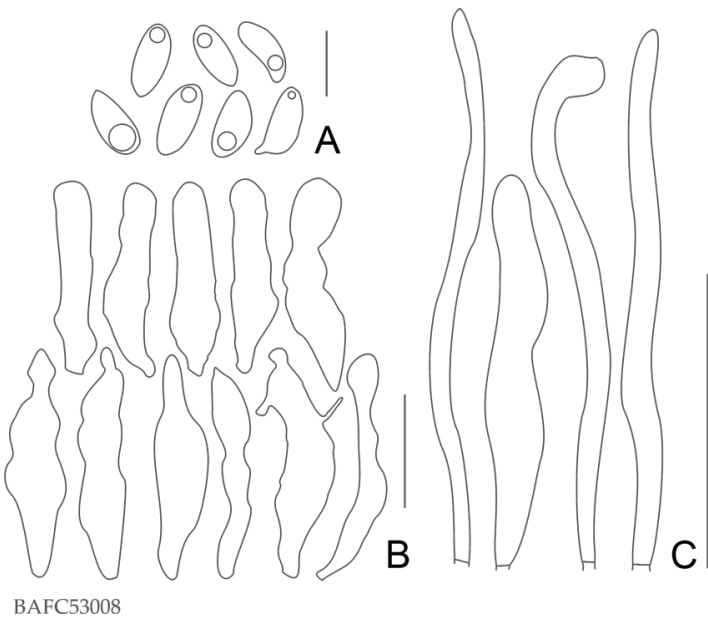

Fig. 7. Collybiopsis subpruinosa. A, esporas (escala: $6 \mu \mathrm{m}$ ). B, queilocistidios (escala: $15 \mu \mathrm{m}$ ). C, caulocistidios (escala: $30 \mu \mathrm{m}$ ). Ilustraciones Agustín P. Martínez.

\section{COMENTARIOS GENERALES}

Las tres nuevas especies registradas en este trabajo se observaron en áreas naturales protegidas y cubiertas por flora exótica implantada por el hombre, lo cual sugiere una introducción mediante esas plantas.

Cruentomycena viscidocruenta se caracteriza por sus basidiomas pequeños, de aspecto micenoide y una coloración rojo intenso muy llamativa. Los primeros registros surgen en Oceanía en 1919, y recientemente se registró su presencia en Sudáfrica (Geesteranus \& Hausknecht, 1998; Grgurinovic, 2002; Kinge et al., 2020), y hasta este trabajo, no se conocían registros de esta especie en otros continentes. El género Cruentomycena se encuentra compuesto por Cr. viscidocruenta y otras dos especies más, Cr. kedrovayae y Cr. orientalis, las cuales se reportaron solamente en Rusia y Corea, y Japón respectivamente (Cho et al., 2018; Petersen et al., 2008; Terashima et al., 2016). Sin embargo, en México, reportaron que Mycena guzmanii se encuentra relacionada filogenéticamente con el género Cruentomycena, dejando en duda si pertenece o no a este género (Cortés-Pérez et al., 2019). Durante más de 28 visitas mensuales realizadas en un periodo de tres años, se observó 
una sola vez la presencia de Cr. viscidocruenta en la reserva Los Robles. Esto sugiere que se trata de una especie con baja frecuencia de ocurrencia en el área de estudio o fructificación esporádica y que no se trate de una inserción reciente en el área, sino que la especie ya se encontraba en el continente Sudamericano, y que debido a su baja frecuencia de aparición y que por poseer basidiomas pequeños no haya sido observado antes.

Por otro lado, Collybiopsis luxurians y $C$. subpruinosa son especies que seguramente ingresaron al país mediante alguna planta exótica. Varios autores afirman que C. luxurians llegó a Norteamérica desde Europa, y con el tiempo se fue desplazando hacia el sur de América (Desjardin et al., 1999; Mata et al., 2006). En particular, se podría haber introducido esta especie mediante ser ejemplares de Casuarina, ya que fue reportada en Hawái dentro de bosques implantados de Casuarina (Desjardin et al., 1999) y también fue hallado bajo los mismos árboles durante este trabajo en Buenos Aires. Collybiopsis subpruinosa es posiblemente una especie autóctona de la Laurisilva de Madeira, la cual luego se desplazó hacia América. Otra especie similar a C. subpruinosa, como Collybiopsis villosipes ya fue reportada en Buenos Aires, Argentina (Lechner, 2015). La presencia y colonización de estas nuevas especies, como C. subpruinosa y $C$. villosipes, podría tomarse como un indicador biológico del cambio climático, ya que son especies reportadas en climas cálidos extendiéndose hacia regiones con climas más templados (Lee et al., 2010; Heilmann-Clausen et al., 2015).

\section{AGRADECIMIENTOS}

Al Consejo Nacional de Investigaciones Científicas y Tecnológicas (CONICET) y la Universidad de Buenos Aires (UBA) por el financiamiento y disponibilidad de equipamiento. Al Dr. Bernardo E. Lechner por su apoyo académico, a Diego Higer por su ayuda bibliográfica, al personal del Parque municipal Los Robles, en especial a Hernán Ibáñez, por su predisposición total en la investigación, a Susana Pereira por su amabilidad, y a C. Grand Martin por todo su apoyo incondicional.

\section{BIBLIOGRAFÍA}

Antonín, V. \& J. Herink. 1999. Notes on the variability of Gymnopus luxurians (Tricholomataceae). Czech Mycology 52(1): 41-49.

Antonín, V. \& M. E. Noordeloos. 2010. A Monograph of Marasmioid and Collybioid Fungi in Europe. IHW Verl. 480 pp. ISBN: 9783930167722.

Bon, M. \& F. Massart. 1996. Deux espèces américaines découvertes dans le sud-ouest de la France. Doc. mycol 26(103): 29-32.

Caffot, M. L. H.; F. Kuhar, M. Gube, E. Crespo \& L. S. Domínguez. 2020. Bovista pezica (Basidiomycota, Agaricales) A new species with unusually ornamented capillitium, from Patagonia Argentina. Nova Hedwigia 111: 173-185.

Cho, H. J.; H. Lee, V. Li, S. Jargalmaa, N. K. Kim, M. J. Kim \& Y. W. Lim. 2018. Six unrecorded macrofungi from the Royal Tombs (Donggureung and Seooreung) of the Joseon Dynasty and Jongmyo Shrine, Korea. Journal of Species Research 7(1): 1-8.

Cleland, S. J. B. \& E. Cheel. 1919. Australian fungi: notes and descriptions, No. 3. sn.

Coimbra, V. R. M. 2017. Riqueza e aspectos moleculares de Gymnopus (Omphalotaceae, Agaricales) do Norte e Nordeste brasileiro. Tesis doctoral Universidad Federal de Pernambuco.

Contu, M. \& S. La Rocca. 1999. Fungi non delineati. Paris IX. Funghi della zona mediterranea insulare italiana. Ed. Mykoflora, Alassio. 48 pp.

Cortés-Pérez, A.; D. E. Desjardin, B. A. Perry, V. RamírezCruz, F. Ramírez-Guillén, A. R. Villalobos-Arámbula \& A. Rockefeller. 2019. New species and records of bioluminescent Mycena from Mexico. Mycologia, 111(2), 319-338.

Desjardin, D. E.; R. E. Halling \& D. E. Hemmes. 1999. Agaricales of the Hawaiian Islands, 5. The genera Rhodocollybia and Gymnopus, Mycologia 91(1): 166-176, DOI: https://doi.org/10.1080/00275514.1999.12061006

Desjardin, D. E.; M. G. Wood \& F. A. Stevens. 2015. California mushrooms: The comprehensive identification guide. Timber Press.

Desjardin, D. E. \& B. A. Perry. 2017. The gymnopoid fungi (Basidiomycota, Agaricales) from the Republic of São Tomé and Príncipe, West Africa. Mycosphere 8: 1317-1391.

González, G. C.; C. Barroetaveña, S. B. Visnovsky, M. Rajchenberg \& M. B. Pildain. 2021. A new species, phylogeny, and a worldwide key of the edible wood decay Fistulina (Agaricales). Mycological Progress 20(5): 733-746. 
Grgurinovic, C. A. \& A. A. Holland. 1982. Macroscopic and Microscopic features of Mycena interrupta and Mycena viscido-cruenta. Brunonia 5(1): 95-101. DOI: https://doi. org/10.1071/BRU9820095

Grgurinovic, C. A. 2002. The genus Mycena in south-eastern Australia. Fungal Diversity Press, Hong Kong.

Halling, R. E. 1983. The genus Collybia in the Northeastern United States and adjacent Canada. Brunswick. Mycol. Memoir 8: 1-148.

Hausknecht, A. \& A. Zuccherelli. 1998. Ritrovamenti interessanti dal Ravennate. 5a parte. Boll. Gruppo Micol. G. Bresadola 41(2): 91-132.

Heilmann-Clausen, J.; E. S. Barron, L. Boddy, A. Dahlberg, G. W. Griffith, J. Nordén, O. Ovaskainen, C. Perini, B. SennIrlet \& P. Halme. 2015. A fungal perspective on conservation biology. Conservation biology 29(1): 61-68.

Kinge, T. R.; G. Goldman, A. Jacobs, G. C. Ndiritu \& M. Gryzenhout. 2020. A first checklist of macrofungi for South Africa. MycoKeys, 63, 1.

Kornerup, A. \& J. H. Wanscher. 1987. Methuen handbook of colour. ISBN 0413334007.

Kuhar, F.; M. E. Smith, A. Mujic, C. Truong \& E. Nouhra. 2017. A systematic overview of Descolea (Agaricales) in the Nothofagaceae forests of Patagonia. Fungal biology 121(10): 876-889.

Largent, D. L. 1986. How to Identify Mushrooms to Genus I Macroscopic Features. Mad River. Eureka, California vol. 1.

Lechner, B. E.; E. Albertó \& R. Petersen. 2003. Pleurotus araucariicola is Pleurotus pulmonarius. Mycotaxon 86 : 425-431.

Lechner, B. E.; J. E. Wright \& E. Albertó. 2004. The genus Pleurotus in Argentina. Mycologia 96(4): 844-857.

Lechner, B. E.; J. E. Wright \& E. Albertó. 2005. The genus Pleurotus in Argentina: mating tests. Sydowia 57(2): 233-245.

Lechner, B. E.; J. E. Wright \& O. Popoff. 2006. New taxa and new records for Argentina of fungi from Iguazú National Park, Misiones. Fungal diversity 21: 131-139.

Lechner, B. E. \& Albertó, E. 2008. Especies tóxicas de Agaricales halladas en la Argentina: nueva cita de Amanita pantherina y reevaluación de la comestibilidad de Tricholoma equestre. Boletin de la Sociedad Argentina de Botánica 43 (3-4): 227-235.

Lechner, B. E. 2015. Species of Agaricales found for the first time in Argentina: Agrocybe molesta, Coprinopsis romagnesiana and Gymnopus villosipes. Boletín de la Sociedad Argentina de Botánica 50(3): 303-307.

Lee, B. Y.; G. H. Nam, J. H. Yun, G. Y. Cho, J. S. Lee, J. H. Kim, T. S. Park, K. Kim \& K. Oh. 2010. Biological indicators to monitor responses against climate change in Korea. Korean Journal of Plant Taxonomy 40(4): 202-207.
Maas Geesteranus, R. A. \& A. Hausknecht. 1998. Two striking Mycenas from the Southern Hemisphere. Österreicische Zeitschrift für Pilzkunde 7: 123-133.

Martínez, A. P.; J. M. Suarez \& B. E. Lechner. 2020. Tres nuevos registros de Agaricomycetes para la Argentina: Cortinarius casimiri var. hoffmannii, Lactarius hepaticus y Mycena margarita. Darwiniana, nueva serie 8(2): 438-448. DOI: https://doi.org/10.14522/darwiniana.2020.82.888

Mata, J. L.; R. E. Halling, K. W. Hughes \& R. H. Petersen. 2004. Rhodocollybia in neotropical montane forests. Mycological Progress 3(4): 337-352. DOI: https://doi.org/10.1007/ s11557-006-0104-x

Mata, J. L.; K. W. Hughes \& R. H. Petersen. 2006. An investigation of Omphalotaceae (Fungi: Euagarics) with emphasis on the genus Gymnopus. Sydowia-Horn 58(2): 191-289.

Mata, J. L. \& C. L. Ovrebo. 2009. New reports and illustrations of Gymnopus for Costa Rica and Panama. Fungal Diversity 38: 125-131.

Murrill, W. A. 1915. Agaricaceae (pars). North American Flora 9(4): 237-255, 286-296.

Niveiro, N. \& E. Albertó. 2012. Checklist of the Argentine Agaricales I. Amanitaceae, Pluteaceae and Hygrophoraceae. Mycotaxon 119: 493-494.

Niveiro, N. \& E. Albertó. 2013. Checklist of the Argentine Agaricales 5. Agaricaceae. Mycotaxon 122(491): 1-25.

Niveiro, N.; O. Popoff, B. E. Lechner \& E. O. Albertó. 2014. Pholiota oblita, new species in sect. Adiposae stirps Subflammans (Strophariaceae, Agaricomycetes), from the Argentinean Yungas. Phytotaxa 167(3): 276-282.

Niveiro, N.; M. Uhart \& E. O. Albertó. 2020. Revision of the genera Agrocybe and Cyclocybe (Strophariaceae, Agaricales, Basidiomycota) in Argentina. Rodriguésia 71: 1-26.

Ortiz, R.; M. Párraga, J. Navarrete, I. Carrasco, E. de la Vega, M. Ortiz, P. Herrera, J. A. Jurgens, B. W. Held \& R. A. Blanchette. 2014. Investigations of Biodeterioration by Fungi in Historic Wooden Churches of Chiloé, Chile. Microbial Ecology 67(3): 568-575. DOI: https://doi. org/10.1007/s00248-013-0358-1

Ovrebo, C. L. 1996. La flora de agáricos (Agaricales) de la Estación Biológica La Selva, Costa Rica. Revista de Biología Tropical 44(4): 39-57.

Petersen, R. H. \& K. W. Hughes. 2007. Some agaric distribution patterns involving Pacific landmasses and Pacific Rim. Mycoscience 48(1): 1-14.

Petersen, R. H.; K. W. Hughes, E. B. Lickey, A. E. Kovalenko, O. V. Morozova \& N. V. Psurtseva. 2008. A new genus, Cruentomycena, with Mycena viscidocruenta as type species. Mycotaxon 105: 119-136. 
A. P. MARTínEZ \& B. E. LECHNER. Nuevos registros de Cruentomycena y Collybiopsis para la Argentina

Petersen, R. H. \& K. W. Hughes. 2021. Collybiopsis and its type species, Co. ramealis. Mycotaxon, 136(2), 263-349.

Rosa, L. H. \& M. Capelari. 2009. Agaricales fungi from Atlantic rain forest fragments in Minas Gerais, Brazil. Brazilian Journal of Microbiology 40(4): 846-851.

Saba, M. \& A. N. Khalid. 2014. New reports of Gymnopus from Pakistan based on ITS sequences. Mycotaxon 129(1): 63-72.

Song, J.; J. F. Liang, M. Mehrabi-Koushki, I. Krisai-Greilhuber, B. Ali, V. K. Bhatt, A. Cerna-Mendoza, B. Chen, Z. X. Chen, H. L. Chu, M. A. Corazon-Guivin, G. A. da Silva, A. De Kesel, B. Dima, F. Dovana, R. Farokhinejad, G. Ferisin, J. C. Guerrero-Abad, T. Guo, L. H. Han, S. Ilyas, A. Justo, A. N. Khalid, S. Khodadadi-Pourarpanahi, T. H. Li, C. Liu, M. Lorenzini, J. K. Lu, A. S. Mumtaz, F. Oehl, X. Y. Pan, V. Papp, W. Qian, A. Razaq, K. C. Semwal, L. Z. Tang, X.
L. Tian, A. Vallejos-Tapullima, N. A. van der Merwe, S. K. Wang, C. Q. Wang, R. H. Yang, F. Yu, G. Zapparoli, M. Zhang, V. Antonín, A. Aptroot, A. Aslan, A. Banerjee, S. Chatterjee, A. C. Dirks, L. Ebrahimi, K. B. Fotouhifar, Y. Ghosta, L. B. Kalinina, D. Karahan, J. Liu, M. K. Maiti, A. Mookherjee, P. S. Nath, B. Panja, J. Saha, H. Ševčíková, H. Voglmayr, K. Yazıcı \& D. Haelewaters. 2019. Fungal Systematics and Evolution: FUSE 5. Sydowia 71: 141-245. DOI: https://doi.org/10.12905/0380.sydowia71-2019-0141

Terashima, Y.; H. Takahashi \& Y. Taneyama. 2016. The fungal flora in southwestern Japan: Agarics and boletes. Tokai Daigaku, Kanagawa, Japan, 349pp.

Wright, J. E. \& E. Albertó. 2002. Guía de hongos de la región pampeana. I. Hongos con laminillas. L.O.L.A., Buenos Aires. 